\title{
SUBCOMPLEXES OF POINCARÉ COMPLEXES
}

\author{
BY J. P. E. HODGSON
}

Communicated by Glen Bredon, March 12, 1974

1. Introduction. This paper is concerned with the following situation. Suppose we are given a simplicial complex $X$ which is a Poincaré duality space for some (twisted) coefficients, and that $K$ is a subcomplex of $X$ which is itself a Poincaré duality space; when can we deduce that the inclusion of $K$ in $X$ is homotopic to a Poincaré embedding?

There are two interesting cases of the above situation, the first is given by a theorem of Bredon [1], which states that if $Z_{p}$ acts on a simplicial complex which is a $Z_{p}$-Poincaré duality space, then each component of the fixed point set is a $Z_{p}$-Poincare duality space. The other case is that of the product of a Poincaré duality space with itself, triangulated so that the diagonal is a subcomplex. We shall give a condition sufficient to obtain an embedding in these two cases, subject to certain dimensional restrictions.

2. Normally embedded subcomplexes. For the purpose of simplicity of exposition, we shall restrict ourselves to the simply-connected case. We shall begin by discussing the case of integer coefficients, and will describe the modifications required for the $Z_{p}$ case when we discuss the Bredon theorem. We assume familiarity with the basic definitions of Poincaré complex, Poincare embedding (they can be found for instance in Levitt [3] ), and with the concept of a normal space and normal pair as defined by Quinn [4] .

It is known [4] that the mapping cylinder of a normal map is a normal pair. Furthermore a Poincare complex is a normal space with the spherical fibration given by the Spivak normal bundle construction.

A Poincare complex of formal dimension $n$, that is presented as an $n$-dimensional simplicial complex, will be called a triangulated Poincaré complex.

AMS (MOS) subject classifications (1970). Primary 57B10, 55F25; Secondary $56 \mathrm{C} 20$.

Key words and phrases. Poincaré duality space, normal space, normally embedded subcomplex, Poincaré embedding. 
Notation. If $G$ is an abelian group, $F G$ will denote the free abelian group obtained by dividing out by the torsion subgroup.

Definition 2.1. Let $X$ be a triangulated Poincaré complex, $K$ a full subcomplex of $X$, and $\nu(k)$ a simplicial neighborhood of $K$ obtained by deriving $X$ near $K$; then $K$ is a normally embedded subcomplex of $X$ if the image of the fundamental class $[X]$ in $F\left(H_{n}(\nu(K)), \partial \nu(K)\right) \cong$ $F\left(H_{n}(X, X-\nu(K))\right)$ induced by $c: X \rightarrow X(X-\nu(K))$ is a generator.

If a group $G$ acts simplicially on $X$ with $K$ an invariant subcomplex, then an obvious modification of the above defines $K$ as an equivariantly normally embedded subcomplex. In the usual way one can also define a normally embedded subpair of a triangulated Poincaré pair.

ExAmple 2.2. Let $P$ be a Poincaré complex of formal dimension $\geqslant 5$; then there is a triangulated Poincaré complex $Q$ homotopy equivalent to $P$ such that $Q \times Q$ can be triangulated so that the diagonal is a normally embedded subcomplex. This follows since $Q$ can be chosen to be a simplicial $n$-cycle (for example by using a handle decomposition [2]).

We note the following lemma.

LEMMA 2.3. Suppose $K$ is a normally embedded subcomplex of a triangulated Poincaré complex $X$, and $f: K \rightarrow L$ a homotopy equivalence, $L$ a simplicial complex. Then $X$ can be modified by a homotopy equivalence so $K$ contains $L$ as a normally embedded subcomplex.

Proof. If $\nu(k)$ is the simplicial neighborhood of $K$, we can excise it and replace it by the mapping cylinder of $\partial \nu(k) \rightarrow \nu(k) \rightarrow K \rightarrow L$.

Corollary. If in Definition 2.1, $K$ is itself a Poincaré complex of formal dimension $\geqslant 5$, we can suppose $K$ is presented as a union of handles.

Proof. According to Jones [2] any Poincaré complex of dimension $\geqslant 5$ has a handle decomposition.

3. The main theorem. The main result of this paper is the following.

Theorem 3.1. Let $X$ be a triangulated Poincaré complex of dimension $n$, and suppose $K$ is a normally embedded subcomplex which is a triangulated Poincaré complex of dimension $k \geqslant 5, k<n$. Then the inclusion $K \rightarrow N$ is homotopic to a Poincaré embedding.

We shall outline the main idea of the proof (details will appear elsewhere 
[6]). Let $\nu(K)$ be the simplicial neighborhood of $K$ in $X$. Suppose we can find a spherical fibration $\xi: E \rightarrow K$ of rank $(n-k) . E$ is then a normal space of formal dimension $(n-1)$. Furthermore one can show that $\partial \nu(K)$ is also a normal space of formal dimension $(n-1)$. So if we can find a normal map $h: \partial \nu(k) \rightarrow E$ we can replace $\nu(K)$ by $M_{h} \cup_{E} M_{\xi}$ in $X$. ( $\left(M_{h}, M_{\xi}\right.$ are the mapping cylinders of $h$ and $\xi$ respectively.) It then follows from Wall [5, Proposition 2.7(ii)] that the pairs $((X-\nu(K)) \cup$ $\left.M_{h}, E\right)$, and $\left(M_{\xi}, E\right)$ give the required splitting of $X$. The construction of $E$ and the normal map $h$ are performed by a fairly straightforward induction using a handle decomposition of $K$.

With a simple modification of the proof, one obtains

Corollary. Let $X$ be a Poincaré complex of formal dimension $n \geqslant 5$ and suppose $X \times X$ is triangulated as a $2 n$-complex so that the diagonal is an equivariantly normally embedded subcomplex, where $Z_{2}$ acts on $X \times X$ by exchange of factors; then there is an equivariant splitting $b: X \times X \rightarrow(M, \partial N) \cup(N, \partial N)$ with $N$ homotopy equivalent to the diagonal and $Z_{2}$ acting freely on $M$. The inclusion $\partial N \rightarrow N$ is homotopic to a spherical fibration, the tangent bundle associated to the given triangulation.

The bundle obtained appears to depend on the triangulation; this will be discussed elsewhere [6].

4. The Bredon theorem. In this section we indicate briefly how our results may be applied to the Bredon theorem mentioned in the introduction. In a manner parallel to Jones [2], we define a category $\mathrm{CW}_{p}$, whose objects are finite $\mathrm{CW}$-complexes and whose morphisms are all continuous maps. A homotopy in $\mathrm{CW}_{p}$ from $h_{1}: X_{1} \rightarrow Y$ to $h_{2}: X_{2} \rightarrow Y$ is a commutative diagram $^{1}$

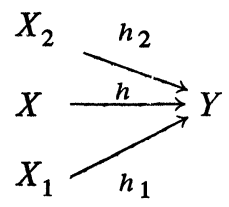

where each vertical map is a $Z_{p}$-homotopy equivalence; that is, an isomorphism of fundamental groups and $Z_{p}$-homotopy. A finite $\mathrm{CW}$-complex $X$ is a

${ }^{1}$ ADDED IN PROOF. In [6] it will be shown that the bundle is independent of the triangulations. 
$\mathrm{CW}_{p}$-Poincaré complex if it satisfies Poincaré duality for $Z_{p}$ coefficients and a $\mathrm{CW}_{p}$-Poincaré embedding is defined in the natural way. $\mathrm{A}_{\mathrm{CW}_{p} \text {-normal }}$ space is a CW-complex $X$ together with a $Z_{p}$-spherical fibration $\xi$ and map $p: S^{n+k} \rightarrow T(\xi)$. As before a simplicial complex of dimension $n$ which is a $\mathrm{CW}_{p}$-Poincaré complex of formal dimension $n$ is called a triangulated $\mathrm{CW}_{p}$. Poincaré complex. Again Jones' work implies that a $\mathrm{CW}_{p}$-Poincaré complex of dimension $\geqslant 5$ has a handle decomposition.

Definition 4.1. Let $X$ be a triangulated $\mathrm{CW}_{p}$-Poincaré complex and $K$ a subcomplex. Then $K$ is a normally embedded subcomplex if $K$ has a simplicial neighborhood $\nu(K)$ in some derived neighborhood of $X$ near $K$ such that the image of the fundamental class $[X] \in H_{n}\left(X, Z_{p}\right)$ under the map $H_{n}\left(X, Z_{p}\right) \rightarrow H_{n}\left(\nu(K), \partial \nu(K) ; Z_{p}\right)$ is nonzero. If $X$ has a group action and $K$ is invariant, then we can define equivariantly normally embedded in the obvious way.

One can then modify the proof of Theorem 3.1 to obtain

Theorem 4.2. Let $X$ be a triangulated $C W_{p}$-Poincaré complex of dimension $n$, and suppose $Z_{p}$ acts simplicially on $X$ with fixed point set $F$. Then for each component $F_{0}$ of $F$ that is a $C W_{p}$-Poincaré complex of formal dimension $\geqslant 5$ and is equivariantly normally embedded; this inclusion $F_{0} \rightarrow X$ is homotopic to an equivariant $C W_{p}$-Poincaré embedding.

To show the applicability of this to the Bredon theorem we need the following results.

Proposition 4.3. Let $X$ be a $C W_{p}$-Poincaré complex of formal dimension $n$, and suppose $Z_{p}$ acts simplicially on $X$ with fixed point sets $F=F_{1} \cup F_{2} \cup \cdots \cup F_{r}$, each $F_{i}$ a $C W_{p}$-Poincaré complex of formal dimension $f_{i} \geqslant 3$. Then the $(r+1)-\operatorname{ad}\left(X ; F_{1}, \cdots, F_{r}\right)$ is homotopy equivalent to an $(r+1)-\operatorname{ad}\left(X^{\prime} ; F_{1}^{\prime}, \cdots, F_{r}^{\prime}\right)$ of triangulated $C W_{p}$-Poincaré complexes.

Proposition 4.4. Let $X$ be a triangulated $C W_{p}$-Poincaré complex of dimension $n$, and suppose $Z_{p}$ acts simplicially on $X$ with fixed point set $F$. Then each component $F_{0}$ of the fixed point set is equivariantly normally embedded.

The first of these propositions is proved by constructing an appropriate model for the quotient space $X / Z_{p}$, and the second uses a spectral sequence argument following the method of proof of Bredon's theorem. We thus obtain 
THEOREM 4.5. Let $X$ be a $C W_{p}$-Poincaré complex represented by a simplicial complex on which $Z_{p}$ acts simplicially. Then if the formal dimension of each component of the fixed point set (as a CW -Poincaré complex) is $\geqslant 5, X$ is equivariantly homotopy equivalent to a triangulated $C W_{p^{-}}$. Poincaré complex $Y$, on which $Z_{p}$ acts and such that the fixed point set is $C W_{p}$-Poincaré embedded.

\section{REFERENCES}

1. G. Bredon, Fixed point sets of actions on Poincaré duality spaces, Topology 12 (1973), 159-176.

2. L. Jones, Patch spaces: A geometric representation for Poincaré spaces, Ann. of Math. (2) 97 (1973), 306-343. MR $47 \# 4269$.

3. N. Levitt, On the structure of Poincaré duality spaces, Topology 7 (1968), 369-388. MR $40 \# 2089$.

4. F. Quinn, Surgery on Poincaré and normal spaces, Bull. Amer. Math. Soc. 78 (1972), 262-267. MR $45 \# 6014$.

5. C. T. C. Wall, Surgery on compact manifolds, Academic Press, New York, 1970.

6. J. P. E. Hodgson, Embedded subcomplexes of Poincaré complexes (in preparation).

\section{DEPARTMENT OF MATHEMATICS, UNIVERSITY OF PENNSYLVANIA, PHILADELPHIA, PENNSYLVANIA 19104}

Current address: Department of Mathematics, Adelphi University, Garden City, Long Island, New York 11530 